\title{
REDISTRIBUTION OF POPULATION AMONG THE HIGHER LEVELS OF Na ATOMS IN AN $\mathrm{H}_{2}-\mathrm{O}_{2}-\mathrm{Ar}$ FLAME
}

\author{
C. A. Van Duk, P. J. Th. Zeegers, and C. Th. J. Alkemade \\ Fysisch Laboratorium, Rijksuniversiteit, P.O. Box 80 000, 3508 TA Utrecht, The Netherlands
}

(Received 1 June 1978)

\begin{abstract}
A substantial fraction of the population of $\mathrm{Na}$-atoms in an $\mathrm{H}_{2}-\mathrm{O}_{2}-\mathrm{Ar}$ flame is found to be distributed among the higher levels as a result of two-photon laser excitation, spontaneous emission and collisions with flame gas atoms and molecules. Several population inversions are observed. From the laser-excited level upwards, a partial Boltzmann equilibrium, corresponding to the flame temperature, has been found in some cases. Estimates of weighted average rate-constants for collisional energy transfer from groups of higher levels to the $3 P$-level are given. Some level-pairs appear to be strongly coupled to each other.
\end{abstract}

\section{INTRODUCTION}

THE STUDY of collisional coupling of atomic levels in flames has concentrated so far on the coupling between the first excited multiplet and the ground state and has resulted in the determination of specific cross sections for collisional transfer for various collision partners. ${ }^{(1,2)}$ In the present experiments, we show the effect of collisional coupling and spontaneous decay on the population of various higher levels of Na-atoms under flame-conditions when one of these levels is populated far above thermal equilibrium by laser irradiation.

When several multiplets are populated, experiments on collisional transfer become more complex for several reasons: (1) The measurement of the intensity of a transition from one level to another arbitrary level is sufficient to determine the population of the first level, provided its Einstein coefficient and the wavelength of the transition are known. The intensities yield the known parameters in the rate equations. The number of collisional rate constants (the unknowns) will in general exceed the number of knowns. (2) Not every level may produce an observable fluorescence line because of the limits of the spectral range and the sensitivity of detection systems. (3) Computer calculations will generally be necessary to solve the rate equations.

We have measured weighted average rate constants. This procedure is also justified because of the partial Boltzmann equilibrium found between level groups in some cases. Extensive experimental material relating to the present article is reported in Ref. (3), not every detail is repeated here. We have rearranged some of the material with emphasis on the resulting population distributions and the coupling between level populations.

\section{EXPERIMENTAL SET-UP}

The experimental set-up is described in detail in Ref. (3). A broadband, flashlamp-pumped tunable dye laser is used; the pulse duration is $1 \mu \mathrm{s}$ and the full width at half maximum (FWHM) of the spectral laser profile is $140 \mathrm{~m} \AA$; this width exceeds known atomic line widths in flames by a factor of two to three. ${ }^{(4)}$ The laser power is several $\mathrm{kW}$ and the laser beam is focused down to a diameter of approx. $100 \mu \mathrm{m}$. The flame is a premixed stoichiometric $\mathrm{H}_{2}-\mathrm{O}_{2}-\mathrm{Ar}$ flame at $1800 \mathrm{~K}$ and atmospheric pressure into which a $2560 \mathrm{ppm} \mathrm{NaCl}$ solution is nebulized. A monochromator fitted with a photomultiplier monitors the fluorescence in the u.v. and the visible region, and a silicon diode in combination with filters detects near-i.r. fluorescence. The spectral response of the former detection system was determined with a calibrated tungsten ribbon lamp and that of the latter was calibrated against a thermopile.

Three different two-photon transitions were excited, as described in Ref. (3). From the resulting fluorescence spectra, we derived three corresponding population distributions, as shown in Fig. 1. These populations are in arbitrary units. When the laser is detuned from a 
two-photon resonance, each population shown, except that of the $3 P$-level, drops below the detection limit; the remaining signal is interpreted as a one-photon excitation of the collisionally broadened wing of the $3 S-3 P$ transition and its value is indicated by an arrow in Fig. 1 . Excitation profiles of the $3 S-4 D$ two-photon transition monitored with the fluorescence signal of the $3 P-3 S$ and $4 P-3 S$ transition are shown in Fig. 2; one-photon wing excitation is evident from a quasicontinuum in the profile of the $3 P-3 S$ signal. A correction for self-absorption was made for the $3 P-3 S$ signal by extrapolating to zero concentration; a curve of growth of the $3 P-3 S$ fluorescence signal with $3 S-4 D$ excitation is shown in Fig. 3. By subtracting the quasi-continuum from the total signal of the $3 P-3 S$ transition, one finds the signal relating to two-photon excitation.

Einstein coefficients were taken from Refs. (5) and (6), which agree reasonably with experimental values. ${ }^{7}$ Since the onset of saturation was observed with each of the two-photon excitation modes, ${ }^{(3)}$ the population of the laser-excited state is expected to be of the same order of magnitude as that of the ground-state.

\section{RESULTS AND DISCUSSION}

A common feature of the population distributions shown in Fig. 1 is the large population of the $3 P$-level, which in each case exceeds the population of the laser-excited state. Obvious

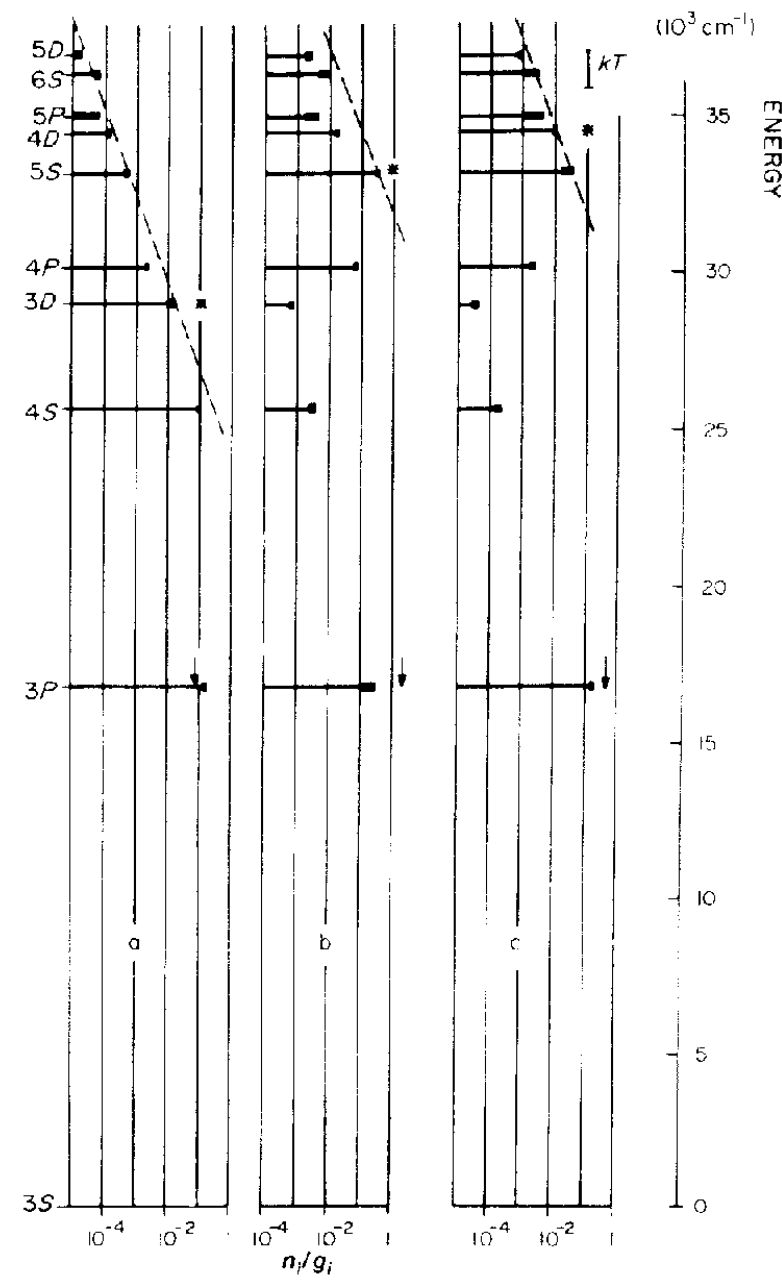

Fig. 1. Level diagram of the Na-atom with relative populations resulting from two-photon excitation indicated by the length of the horizontal level bars; blobs indicate statistical error limits. The $M_{j}$ levels of a doublet (doublet separation $\& k T$ ) are assumed to be equally populated by fast mixing collisions; the depicted populations $n_{i}$ divided by the statistical weight of the doublet $g_{i}$ thus pertain to those of a single $M$ s level and are plotted along the horizontal (logarithmic) scale. The $3 P$ population resulting from one-photon wing excitation (see text) is indicated with an arrow. Slanted lines correspond to Boltzmann equilibrium at the flame temperature. Asterisks mark the laser-excited level: (a) $3 S-3 D$ excitation; (b) $3 S-5 S$ excitation; (c) $3 S-4 D$ excitation. 


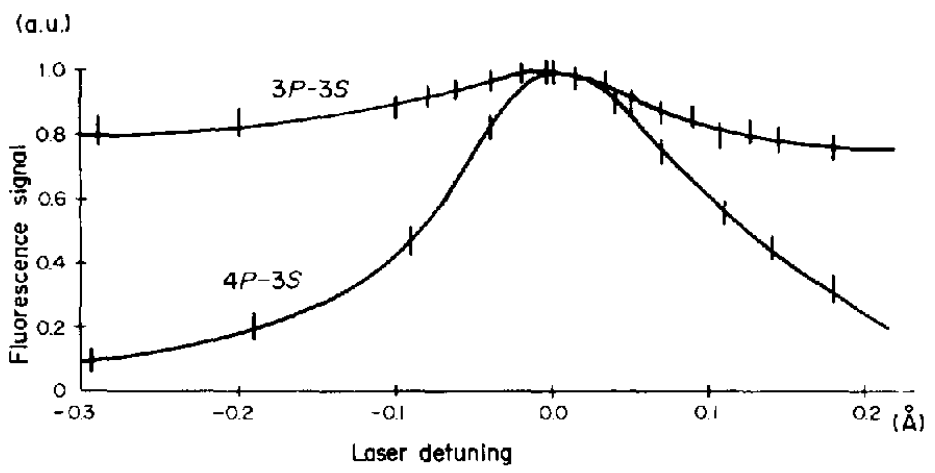

Fig. 2. Fluorescence signals as a function of the laser tuning across the $3 S-4 D$ two-photon transition. The lower curve corresponds to the $4 P-3 S$ signal and the upper curve belongs to the $3 P-3 S$ signal, the quasi-continuum in the latter curve is interpreted as the result from one-photon excitation of the collisionally broadened wing of the $3 P-3 S$ transition.

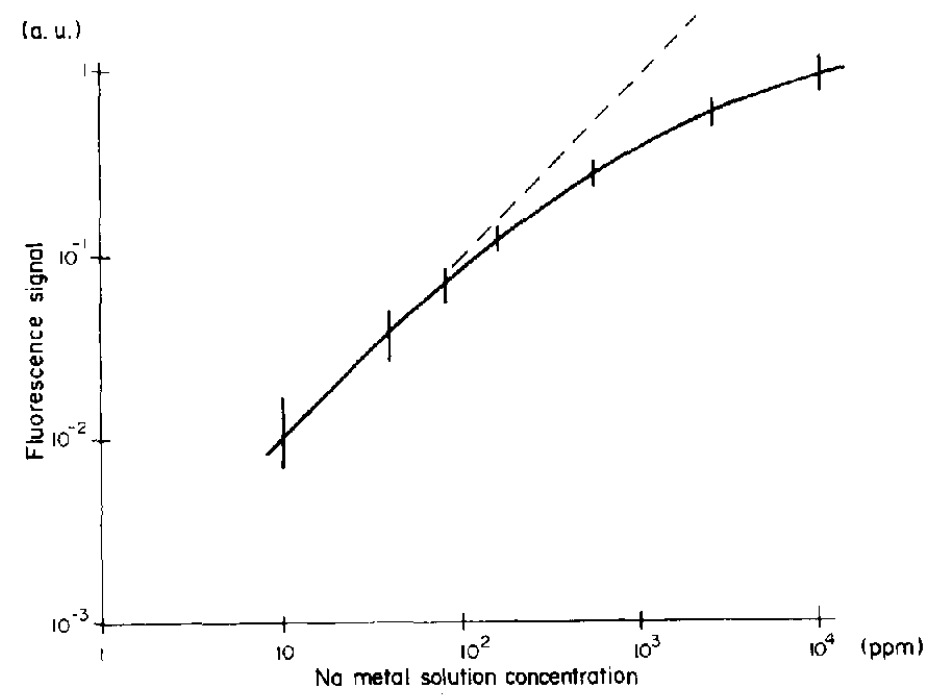

Fig. 3. Fluorescence signal of the $3 P-3 S$ transition as a function of the Na-metal solution concentration. The laser was tuned to the $3 S-4 D$ two-photon transition. The two-photon excitation experiments described in this paper were done at a Na-concentration of $2560 \mathrm{ppm}$.

inversions occur between $S$-states and between $D$-states in the cases of $3 S-4 D$ and $3 S-5 S$ two-photon excitation. Inversions in alkali vapours have been predicted for Rydberg states by LAU et al. ${ }^{(8)}$ and observed by KUNG et al. ${ }^{(9)}$ Also, single-pass, stimulated, collision-induced fluorescence (SCF) in a thallium-argon mixture was observed by CARLSTEN and RAYMER ${ }^{(10)}$ who report an increase in SCF with increasing Ar pressure. Table 1 lists the observed population ratios $n_{i} g_{j} /\left(n_{i} g_{i}\right)$, where $g_{i} / g_{j}$ and $n_{i} / n_{i}$ are the ratios of the statistical weights and of the populations of the doublets $i$ and $j$, respectively. Collisional doublet mixing is assumed to be sufficiently fast to justify the summation of the statistical weights and populations of the doublet components. ${ }^{(11-14)}$ The experimental scatter in these population ratios amounts to about $30 \%$. Fifteen inversions occur which clearly exceed the experimental error; eight inversions are between radiatively allowed transitions and have wavelengths in the i.r. region. The magnitude of the inversions increases with increasing energy of the laser-excited level.

Levels above the laser-excited level are markedly less populated and tend to be in partial Boltzmann equilibrium with each other. This is quantitatively shown in Table 2 , where values are given for the quantity

$$
R_{i j} \equiv \frac{n_{i} / g_{i}}{n_{j} / g_{j}} \mathrm{e}^{\left(E_{i}-E_{j}\right) / k T}
$$

which measures the deviation from partial Boltzmann equilibrium of the observed population 
Table I. Weighted population ratios $n_{i} g_{i}\left(n_{j} g_{i}\right)$ derived from the fluorescence spectra of Ref. (3): Levels $i$ and $j$ are indicated at the top and the left-hand side of the table, respectively, in the order of their excitation energy. The population of the $3 P$-level includes the contribution of one-photon wing excitation (see text). From each set of three numbers, the upper number corresponds to $3 S-3 D$ excitation, followed by that for $3 S-5 S$ excitation and the bottom one refers to $3 S-4 D$ excitation. Numbers in parentheses are prwers of ten.

\begin{tabular}{|c|c|c|c|c|c|c|c|}
\hline $5 b$ & ts & $\mathrm{SP}^{\mathrm{P}}$ & 41.1 & is & it & 33 & $4 \mathrm{~s}$ \\
\hline $\begin{array}{ll}2.9 & (-1) \\
2.5 & (-1) \\
3.4 & (-1)\end{array}$ & & & & & & & \\
\hline $\begin{array}{l}6.7(-1) \\
2.8(-1) \\
\end{array}$ & $\begin{array}{r}2.7(0) \\
8.3(-1) \\
\end{array}$ & & & & & & \\
\hline $\begin{array}{l}1.2(-1) \\
1.1(-1) \\
6.1(-2)\end{array}$ & $\begin{array}{l}4.0(-1) \\
4.4(-1) \\
1.8(-1)\end{array}$ & $\begin{array}{l}1.7(-1) \\
2.2(-1)\end{array}$ & & & & & \\
\hline $\begin{array}{ll}3.0 & (-2) \\
7.1 & (-3) \\
2.8 & (-2)\end{array}$ & $\begin{array}{l}1.0(-1) \\
2.9(-2) \\
8.2(-2)\end{array}$ & $\begin{array}{ll}\ldots & \\
1.1 & (-2) \\
9.8 & (-2)\end{array}$ & $\begin{array}{ll}2.6 & (-1) \\
6.4 & (-2) \\
4.6 & (-1)\end{array}$ & & & & \\
\hline $\begin{array}{ll}7.0 & (-3) \\
3.4 & (-2) \\
3.3 & (-1) \\
\end{array}$ & $\begin{array}{rr}2.4(-2) \\
1.4(-1) \\
9.8(-1) \\
\end{array}$ & $\begin{array}{ll}\cdots & \\
\cdots & (-2) \\
1.2 & (0) \\
\end{array}$ & $\begin{array}{cc}6.0 & (-2) \\
3.1 & (-1) \\
3.4 & (0) \\
\end{array}$ & $\begin{array}{l}\therefore 3(-1) \\
4.85(1) \\
1.2 \quad(+1)\end{array}$ & & & \\
\hline $\begin{array}{ll}1.1 & (-3) \\
3.2 & (0) \\
1.8 & (0)\end{array}$ & $\begin{array}{l}3.7(-3) \\
1.3(+1) \\
3.3(+1)\end{array}$ & $\begin{array}{ll}3.8 & (0) \\
4.8 & (+1)\end{array}$ & $\begin{array}{ll}9.2 & \{-3\} \\
29 & (+1) \\
2.9 & (+2)\end{array}$ & $\begin{array}{ll}3.5 & (-2) \\
4.5 & (+2) \\
6.5 & (+2)\end{array}$ & $\begin{array}{l}1 .(-1) \\
4.4(+1) \\
5 .(+1)\end{array}$ & & \\
\hline $\begin{array}{l}1.3(-4) \\
\text { b.2 }(-1) \\
3.7(0)\end{array}$ & $\begin{array}{ll}3.2 & (-4) \\
3.5 & (0) \\
1.1 & (+1)\end{array}$ & $\begin{array}{l}9.3(-1) \\
1.3(+1)\end{array}$ & $\begin{array}{l}1.3(-3) \\
5.3(0) \\
6.1(+1)\end{array}$ & $\begin{array}{l}4.9(-3) \\
8.6(+1) \\
1.3(+2)\end{array}$ & $\begin{array}{ll}2 . & -1\} \\
1.8 & +1\} \\
1.2 & +11\end{array}$ & $\begin{array}{l}1.4(-1) \\
1.9(-1) \\
\therefore .1\end{array}$ & \\
\hline $\begin{array}{ll}7.8 & (-5) \\
5.2 & (-4) \\
6.8 & (-4)\end{array}$ & $\begin{array}{l}2.7(-4) \\
2.1(-3) \\
\therefore .0(-3)\end{array}$ & $\begin{array}{ll}7.3 & (-4) \\
2.4 & -3)\end{array}$ & $\begin{array}{ll}4.7 & (-4) \\
4.8 & (-3) \\
1.0 & (-2)\end{array}$ & $\begin{array}{ll}2.5 & (-3) \\
7.4 & (-2) \\
2.4 & (-2)\end{array}$ & $\begin{array}{ll}\therefore & (-2) \\
1 & -2 \\
\therefore & -3\end{array}$ & $\begin{array}{ll}7.3 & (-2) \\
1,+6 & (-6) \\
3.7 & (-5)\end{array}$ & $\begin{array}{ll}5.2 & (-1) \\
8.3 & (-4) \\
1.8 & -4\end{array}$ \\
\hline
\end{tabular}

Table 2. Listed numbers are the experimental values of $R_{\mathrm{j}}$, which is defined in the text as a measure for the deviation of the population ratio of levels $i$ and $j$ from partial Boltzmann equilibrium at the flame temperature. Levels $i$ and $j$ are indicated at the top and the left-hand side of the table, respectively, in order of their excitation energy. The population of the $3 P$-level includes the contribution of one-photon wing excitation (see text). From each set of three numbers the upper number corresponds to $3 S-3 D$ excitation, followed by that for $3 S-5 S$ excitation and the bottom one refers to $3 S-4 D$ excitation. Numbers in parentheses are powers of ten.

\begin{tabular}{|c|c|c|c|c|c|c|c|c|}
\hline & 50 & $6 \mathrm{~S}$ & $S P$ & 40 & $3 \mathrm{~s}$ & $4 \mathrm{P}$ & $3 \mathrm{D}$ & $4 \mathrm{~S}$ \\
\hline $6 \mathrm{~S}$ & $\begin{array}{l}4.9(-1) \\
4.2(-1) \\
5.8(-1)\end{array}$ & & & & & & & \\
\hline $5 P$ & $\begin{array}{ll}3.3 & (0) \\
1.4 & (0)\end{array}$ & $\begin{array}{ll}7.7 & (0) \\
2.4 & (0)\end{array}$ & & & & & & \\
\hline $4 \mathrm{D}$ & $\begin{array}{ll}8.5 & (-1) \\
8.1 & (-1) \\
4.5 & (-1)\end{array}$ & $\begin{array}{rr}1.7 & (0) \\
1.9 & (0) \\
7.7 & (-1)\end{array}$ & $\begin{array}{l}\ldots \\
2.5(-1) \\
3.2(-1)\end{array}$ & & & & & \\
\hline $5 \mathrm{~s}$ & $\begin{array}{ll}6.5 & (-1) \\
1.5 & (-1) \\
6.0 & (-1)\end{array}$ & $\begin{array}{lr}1.3 & (0) \\
3.6 & (-1) \\
1.0 & (0)\end{array}$ & $\begin{array}{ll}\ldots & \\
4.7 & (-2) \\
4.3 & (-1)\end{array}$ & 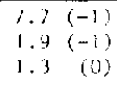 & & & & \\
\hline $4 \mathrm{P}$ & $\begin{array}{rr}1.6 & (0) \\
7.7 & (0) \\
7.4 & (+1)\end{array}$ & $\begin{array}{l}3.2(0) \\
1.8(+1) \\
1.3(+2)\end{array}$ & $\begin{array}{l}2.3(0) \\
5.4(+1)\end{array}$ & $\begin{array}{rr}1.8 & (0) \\
9.4 & (0) \\
1.7 & (+2)\end{array}$ & $\begin{array}{lr}2.4 & (0) \\
5.0 & (+1) \\
1.2 & (+2)\end{array}$ & & & \\
\hline 30) & $\begin{array}{l}5.8(-1) \\
1.7(+3) \\
9.6(+3)\end{array}$ & $\begin{array}{lr}1.2 & (0) \\
4.1 & (+3) \\
1.7 & (+4)\end{array}$ & $\begin{array}{l}5.3(+2) \\
6.9(+3)\end{array}$ & $\begin{array}{ll}6.8(-1) \\
2.1(+3) \\
2.2(+4)\end{array}$ & $\begin{array}{ll}8.9 & (-1) \\
1.1 & (+4) \\
1.6 & (+4)\end{array}$ & $\begin{array}{ll}3.7 & (-1) \\
2.3 & (+2) \\
1.3 & (+2)\end{array}$ & & \\
\hline $4 \mathrm{~S}$ & $\begin{array}{l}1.3(0) \\
5.1(+3) \\
3.1(+4)\end{array}$ & $\begin{array}{ll}2.6 & (0) \\
1.2 & (+4) \\
5.4 & (+4)\end{array}$ & $\begin{array}{ll}\ldots & \\
1.6 & (+3) \\
2.2 & (+4)\end{array}$ & $\begin{array}{rr}1.5 & (0) \\
h .3 & (+3) \\
6.9 & (+4)\end{array}$ & $\begin{array}{l}1.9 \\
3.4(0) \\
3.2(+4) \\
3.2(+4)\end{array}$ & $\begin{array}{ll}8.0 & (-1) \\
6.7 & (+2) \\
4.1 & (+2)\end{array}$ & $\begin{array}{ll}2.2 & (0) \\
3.0 & (0) \\
3.2 & (0)\end{array}$ & \\
\hline $3 P$ & $\begin{array}{ll}7.3(+2) \\
4.8(+3) \\
6.3(+3)\end{array}$ & $\begin{array}{ll}1.5 & (+3) \\
1.1 & (+4) \\
1.1 & (+4)\end{array}$ & $\begin{array}{ll}\cdots & (+3) \\
4.7 & (+3)\end{array}$ & $\begin{array}{ll}8.6 & (+2) \\
6.1 & (+3) \\
1.4 & (+4)\end{array}$ & $\begin{array}{ll}1.1 & (+3) \\
3.2 & (+4) \\
1.1 & (+4)\end{array}$ & $\begin{array}{ll}4.6 & (+2) \\
5.5 & (+2) \\
8.5 & (+1)\end{array}$ & $\begin{array}{lr}1.3 & (+3) \\
2.8 & (0) \\
0.5 & (-1)\end{array}$ & $\begin{array}{ll}5.8(+2) \\
9.6(-1) \\
2.0(-1)\end{array}$ \\
\hline
\end{tabular}


ratio $n_{i} g_{j} /\left(n_{j} g_{i}\right)$; here $T=$ flame temperature $(1800 \mathrm{~K}) ; k=$ Boltzmann's constant; $E_{i}-E_{j}=$ energy difference between levels $i$ and $j$. With each of the excitation modes, the populations of the $4 D$ - and $5 D$-levels are approximately in equilibrium with each other. Similarly, there is partial equilibrium between the $5 S$ - and $6 S$-level, between the $5 D$ - and $6 S$-level and, to a lesser extent, between the $5 S$ - and $5 D$-level. Large deviations occur when the $3 D$-, $4 S$ - or $3 P$-level is involved.

The approach to Boltzmann equilibrium within the group of levels above the $3 P$-level in the case of $3 S-3 D$ excitation and within the group of levels above the $4 P$-level in the case of $3 S-4 D$ excitation presents an additional difficulty for the determination of collisional rate constants between levels within these groups. The reason is that, in an equilibrated level system, the rates of each endo-ergic and reverse exo-ergic transition are in detailed-balance; only if an external disturbance unbalances these rates, can we determine, in principle, the corresponding rate constants. Levels of such an equilibrated group of levels cannot have a coupling constant with an "outside" level which significantly exceeds its maximum coupling constant with the "inside" levels, because in this case the inside level would be drained. Therefore, we assume the coupling of equilibrated level groups with other levels to be rather weak and we characterize this coupling by a weighted average coupling constant $G$. Thus, the system of Fig. 1(a) is reduced to a three-level system consisting of the $3 S$-level, the $3 P$-level and the group of levels from $4 S$ upwards; this procedure gives for the steady state of the $3 P$-level the balance equation

$$
\sum_{i=4 S}^{S D}\left(G_{1}+A_{i-3 P}\right) n_{i}=(A+k) n_{3 P}-\left(\Gamma_{w^{\prime}}+\Gamma_{s}\right) n_{3 S}
$$

where $G_{1}=$ weighted average collisional rate constant for transitions from the group of levels from $4 S$ upwards to the $3 P$-level for the case of $3 S-3 D$ excitation; $A_{i-3 P}=$ Einstein coefficient for spontaneous emission from level $i$ to level 3P;A= Einstein coefficient for spontaneous emission of the $3 P-3 S$ transition; $k=$ quenching rate constant for the $3 P$-level; $\Gamma_{w}=$ transition probability per second for radiative excitation in the collisionally broadened wing of the $3 S-3 P$ transition; $\Gamma_{s}=$ transition probability per second for excitation of the $3 P$-level due to reabsorption of spontaneously emitted photons. In the summation, we assume that all levels above the $4 S$-level are equilibrated between each other, in particular the $4 F$ - and $5 F$-levels are assumed to be in equilibrium with the other members of the group, as these $F$-levels are at a distance of less than $100 \mathrm{~cm}^{-1}$ from the $4 D$ - and $5 D$-levels $\left(k T \wedge 1200 \mathrm{~cm}^{-1}\right)$ and cross sections for mixing between $D$-states and $l>2$ states of sodium atoms colliding with argon atoms are of the order of $10^{3} \AA^{2}$.(15)

With the laser detuned from the two-photon resonance but still at practically the same position in the collisional wing of the $3 S-3 P$ transition, the only remaining fluorescence signal is that ensuing from one-photon wing excitation. In this case, one has

$$
(A+k) n_{3 P}^{\prime}=\Gamma n_{3 S}^{\prime}
$$

where $\Gamma \equiv \Gamma_{w}+\Gamma_{s} ; n_{3 P}^{\prime}$ and $n_{3 S}^{\prime}$ denote the populations of the $3 P$ and $3 S$ levels, respectively, resulting from wing excitation only. In a forthcoming paper dealing with ionization, $\dagger$ we shall present experimental evidence that the degree of ionization is negligible when the laser is resonant with the $3 S-3 P$ one-photon transition or the $3 S-5 S$ two-photon transition for the Na-metal concentrations used. For this reason, we assume that the number density of sodium atoms is virtually constant whether or not the laser is tuned on a two-photon resonance; we therefore have

$$
\sum_{i=4 S}^{S D} n_{i}+n_{3 P}+n_{3 S}=n_{3 P}^{\prime}+n_{3 S}^{\prime}
$$


We eliminate the ground-state densities $n_{3 S}$ and $n_{3 S}$ from Eqs. (1)-(3) and obtain

$$
G_{1}=(A+k+\Gamma) \frac{n_{3 P}}{\sum n_{i}}\left(1-\frac{n_{3 P}^{\prime}}{n_{3 P}}\right)+\Gamma \cdots \frac{\sum A_{i} n_{i}}{\sum n_{i}} .
$$

where we have written $\Sigma$ for $\sum_{i=4 S}^{5 D}$.

In the derivation of Eq. (4), we assume that the stationary state is reached within the laser pulse; this assumption is justified by the magnitude of the known rate constant $(A+k)$ and of the rate constants found in this work. We also assume that the rate constants for collisional transitions from the $3 S$ - and $3 P$-level to the $4 S$ - and higher levels are negligible; this conjecture can easily be justified by the appreciable endo-ergicity of these collisions.

When we compare the population rate of the $3 P$-level caused by the reabsorption of spontaneously emitted radiation with the population rate produced by one-photon wing excitation in a cylindrical volume of radius $r$ (i.e. the flame volume irradiated by the laser beam), we obtain $\Gamma_{s} / \Gamma_{w}=\overline{k(\lambda)} r Y$, as shown in the appendix; $Y$ is the efficiency of Na-D fluorescence for our flame ${ }^{(1)}$ and $k(\lambda)=$ absorption coefficient of the $3 P-3 S$ transition, as averaged over the absorption line profile. As shown in the appendix, $\Gamma_{s}$ is estimated to be $0.1 \Gamma_{w} \cdot \Gamma_{w}$ can be estimated within a factor of two from the known laser irradiance and the value of the absorption coefficient in the wing of the $3 S-3 P$ transition at the laser wavelength; ${ }^{(16)}$ we find $0.8 \times 10^{6} \mathrm{~s}^{-1} \leqslant \Gamma_{\mathrm{w}} \leqslant 3 \times 10^{6} \mathrm{~s}^{-1}$. The value of $A+k=(9.0 \pm 0.05) \times 10^{7} \mathrm{~s}^{1}$ is found from the fluorescence efficiency measurements of LuNsE ${ }^{(1)}$ for our flame $(=$ his flame No. 20). We finally obtain $G_{1}=(2.4 \pm 0.9) \times 10^{8} \mathrm{~s}^{-1}$,

For $3 S-4 D$ excitation, the group of levels above the $4 P$-level is nearly equilibrated and we again replace the level system by a three-level system, this time consisting of the $3 S$-level, the $3 P$-level and the group of levels from $5 S$ upwards. The $4 S-, 3 D$-, and $4 P$-levels are neglected because an improbably large rate constant is needed to make the rates of these levels comparable to the rates of the other levels. Analogous to Eqs. (1)-(3), we obtain

$$
G_{2}=(A+k+\Gamma) \frac{n_{3 P}}{\sum n_{i}}\left(1-\frac{n_{3 P}^{\prime}}{n_{3 p}}\right)+\Gamma-\frac{\sum A_{i} n_{i}}{\sum n_{i}},
$$

where $G_{2}=$ weighted average rate constant of collisional transitions from this group of levels to the $3 P$-level in the case of $3 S-4 D$ excitation, and where we have written $\Sigma$ for $\sum_{i=5 S}^{5 D}$. By inserting the appropriate values, one obtains: $G_{2}=(3 \pm 1) \times 10^{8} \mathrm{~s}^{-1}$, which is of the same order as the estimate for $G_{1}$.

The magnitudes of $G_{1}$ and $G_{2}$ exceed the Einstein coefficients for spontaneous emission by at least a factor five and the quenching rate constant of the $3 P$-level by almost one order of magnitude. ${ }^{(1)}$ The latter fact might be indicative of a more general trend, namely, that collisional rate constants increase with increasing principal quantum number for a given alkali atom.

The gases of our flame are composed of $83 \%$ Ar and $17 \% \mathrm{H}_{2} \mathrm{O}$. The quenching of the first resonance doublet of $\mathrm{Na}$ is almost entirely due to $\mathrm{H}_{2} \mathrm{O}^{(1)}$ but, for the quenching of higher levels, the presence of Ar might be detectable, since theoretical work done by PASCALE and VANDE. PLANQUE ${ }^{(17)}$ shows a number of pseudo-crossings between the potential curves of higher levels of alkali-rare-gas systems. At present, however, no theoretical estimates of cross sections for collisional transitions between higher excited states are available. In this connection, we draw attention to the results of GounAND et al. ${ }^{(18)}$ who claim to have measured a cross section of $8 \AA^{2}$ for the quenching of the $K(10 P)$-state by argon; argon is, however, known to have negligible cross sections for quenching of the first resonance doublet of the alkalis. ${ }^{(19,20)}$ Other experimenters have also reported that various perturbers show increased quenching efficiency on the higher levels of alkali atoms compared with the quenching of the first resonance doublet. ${ }^{(21-24)}$ GALLAGHER et al. ${ }^{(25)}$ report cross sections for quenching of the $5 S$ - and $4 P$-states of $\mathrm{Na}$ by $\mathrm{N}_{2}$, which are two to four times larger than those found by LuNsE ${ }^{(1)}$ for quenching of the $3 P$-state of 
$\mathrm{Na}$ by $\mathrm{N}_{2}$. Gallagher et al ${ }^{(15)}$ suggest, however, that the cross sections for quenching of the higher excited states by rare gases are below $1 \AA^{2}$.

For $3 S-5 S$ excitation, the approach to partial Boltzmann equilibrium is less pronounced. This result may be explained as follows. If the ratio of the coupling constant between the $4 D$ and $5 S$-level to the coupling constant with all lower levels is small for the $4 D$-level but large for the $5 S$-level, then $n_{4 D} / n_{5 S}$ will be closer to the Boltzmann ratio in the case of $4 D$ excitation than in the case of $5 S$ excitation.

As can be seen from Table 1 , the ratios $n_{5 D} / n_{6 S}, n_{6 S} / n_{4 D}, n_{5 D} / n_{4 D}$ and $n_{3 D} / n_{4 S}$ remain approximately constant with various modes of excitation. Strong collisional coupling between the members of each pair thus exists.

Pulse-to-pulse variations of the laser light, r.f. noise from the spark gap which fires the flashlamp, irregularities of the sprayer performance, and fluctuations in the gas supply tend to depress the signal-to-noise ratio. There are also gradual effects from the degrading of the dye solution, drift of the laser tuning and changes in sprayer efficiency. These factors severely limit the precision of the measurements. The low repetition rate of the laser $(1 \mathrm{~Hz})$ makes the measurements very time consuming. The mean value of the experimental scatter in the relative populations plotted in Fig. 1 amounts to about $20 \%$.

With either $G_{1}$ or $G_{2}$, the value of $\Gamma$ is one order of magnitude below that of $A+k$ and errors in $\Gamma$ will hardly affect the error in $G_{i} . A+k$ was measured by LINSE ${ }^{(1)}$ within $5 \%$; we repeated his measurements and found the same value within an error margin of $10 \%$. The quantities $n_{3 p} / \Sigma n_{i}$ and $\Sigma A_{i} n_{i} / \Sigma n_{i}$ were calculated from the measured $n_{i}$ and known $A_{i} ;{ }^{(3,6)}$ we found $n_{3 P} / \Sigma n_{i}=3.2 \pm 1.1, \Sigma A_{i} n_{i} / \Sigma n_{i}=(3.3 \pm 0.7) \times 10^{7} \mathrm{~s}^{-1}$ in the case of $3 S-3 D$ excitation and $n_{3 \mathrm{P}} / \Sigma n_{i}=17 \pm 5, \Sigma A_{i} n_{i} / \Sigma n_{i}=(4.8 \pm 0.9) \times 10^{6} \mathrm{~s}^{-1}$ in the case of $3 S-4 D$ excitation. The magnitudes of the term $\Sigma A_{i} n_{i} / \Sigma n_{i}$ are thus seen to be one order of magnitude below that of the first term with both $3 S-3 D$ and $3 S-4 D$ excitation. When $n_{3 P}^{\prime} / n_{3 P}$ is close to unity, this quantity becomes the main source of error; consequently, we measured $n_{3 P}^{\prime} / n_{3 P}$ with increased precision in the case of $3 S-4 D$ excitation and we found $0.82 \pm 0.06$. In the case of $3 S-3 D$ excitation, $n_{3 P}^{\prime} / n_{3 P}=0.07 \pm 0.02$.

\section{CONCLUSION}

With two-photon excitation processes and laser powers of the order of a few $\mathrm{kW}$, it is possible to create population inversions between some levels of $\mathrm{Na}$-atoms in an $\mathrm{H}_{2}-\mathrm{O}_{2}-\mathrm{Ar}$ flame of $1 \mathrm{~atm}$. The highest populations are found in levels around the laser-excited level; from approximately this level upwards, a partial Boltzmann equilibrium predominates in some cases, inhibiting the determination of rate constants between these equilibrated levels.

The values of the weighted average rate constants $G_{1}$ and $G_{2}$ relating to collisional transfer from groups of higher levels to the $3 P$-level are at least five times higher than the Einstein coefficients for spontaneous transitions from higher levels. $G_{1}$ and $G_{2}$ exceed the quenching rate constant of the first resonance doublet by almost one order of magnitude.

Because $G_{1} \simeq G_{2}$, the dominant levels of either group (i.e. the $4 S$ - and $3 D$-level with $3 S-3 D$ excitation and the $5 S$ - and $4 D$-level with $3 S-4 D$ excitation, see Fig. 1) are likely to have rate constants of the same order of magnitude for collisional transitions to the $3 P$-level.

The assumption of a steady-state population produced by a laser pulse of $1 \mu \mathrm{s}$ seems justified since $G_{1}$ and $G_{2}$ are of the order of $10^{8} \mathrm{~s}^{-1}$ and at least some of the coupling constants within an equilibrated group are likely to be even larger. Specially strong coupling has been found between the members of the level pairs: $(5 D, 6 S),(6 S, 4 D)$, and $(5 D, 4 D)$, because the levels of each pair maintain an almost fixed population ratio with different excitation modi.

Acknowledgement-The authors wish to thank Miss S. M. MCNAB M. A. for linguistic assistance.

\section{REFERENCES}

1. P. I. LIJNSE and R. J. ElsENAAR, JQSRT 12, 1115 (1972).

2. P. L. LIJNSE and C. J. VAN DER MAAS, JQSRT 13, 741 (1973).

3. C. A. Van Duk, P. J. Th. Zeegers, G. Nienhuis, and C. TH. J. Alkemade, JOSRT 20, 55 (1978).

4. W. BEHMENBURG, JQSRT 4, 177 (1964).

5. O. S. Heavens, J. Opt. Soc. Am. 51, 1058 (1961). 
6. W. HAPPER and P. TSEKERIS, Columbia University, New York; unpublished work, with kind permission of the authors.

7. T. F. Gallagher, S. A. Edelstein, and R. M. Hill, Phys. Rev. A16, 2360 (1976).

8. A. M. F. Lal, W. K. Bishel, C. K. Rhodes, and R. M. Hill, Appl. Phys. Lett. 29, 245 (1976).

9. R. T. V. KUNG and I. ITZKAN, IEEE J. QE QE-13, 73 (1977).

10. J. L. CARLSTEN and M. G. RaYMER, Laser Spectroscopy-III, p. 205. Proc. Third Int. Conf. Wyoming, Springer Verlag. Berlin (1977).

11. J. Pitre and L. Krause, Can. J. Phys. 45, 2671 (1967).

12. P. L. Lijse. P. J. Th. ZeEgers, and C. TH. J. AlKemade, JQSRT 13, 1033 (1973).

13. P. L. Lunse, P. J. Th. ZeEgers, and C. Th. J. AlKemade, JQSRT 13. 1301 (1973).

14. P. L. LIINSE and J. C. HoRnMaN, JQSRT 14, 1079 (1974).

15. 'T. F. Gall.agher, S. A. Edelstein, and R. M. Hil. L, Phys. Rev. Lett. 35, 644 (1975).

16. C. A. Vax DiJK, Opt. Comm. 22, 343 (1977).

17. J. Pascale and J. Vandeplanoue, J. Chem. Phys. 60. 2278 (1973).

18. F. Goljnand, J. Cuvellier, P. R. Fournier, and J. Berlande. J. de Phys. 37, L-169 (1976).

19. M. G. Edwakds, J. Phys. B2, 719 (1969).

20. C. Th. J. Alkemade and P. J. Th. ZeEgers, Spectrochemical Methods of Analysis (Edited by J. D. WNEFORDNER). Chap. I, p. 48. Wiley, New York (1971).

21. I. N. Siara and L. Krause, Can. J. Phys, 51, 257 (1973).

22. B. L. Earl and R. R. Herm, Chem. Phys. Lett. 22, 95 (1973).

23. J. Cuvfil..jer, P. R. Fournier, F. Gounand, J. Pascale, and J. Berlande, Phys. Rev. Alt, 846 (1975).

24. M. LuKaszEwicZ, Bull. de l'Acad. Polonaise des Sciences XXIIl, 501 (1975).

25. T. F. Gallaghfr, W. E. Cooke, and S. A. Edelstein, Phys. Rev. A17, 125 (1978).

26. С. TH, J. Alkemade and P. J. TH. ZeEgers, Spectrochemical Methods of Analysis (Edited by J. D. WineFordner), Chap. I, p. 18. Wiley, New York (1971)

\section{APPENDIX}

Since the Na-atom densities used in the two-photon experiment are of the order of $10^{12} \mathrm{~cm}^{-3}$ and self-absorption of the $3 P-3 S$ fluorescence light is known to occur at these densities, it is desirable to have an estimate of the amount of $3 S-3 P$ excitation caused by reabsorption of these fluorescence photons.

In the following, we consider a volume $V$ within the flame which is irradiated by an external source. We derived an expression for the transition probability per second $\Gamma_{s}$ for the reabsorption of spontaneous emission in terms of the transition probability per second $\Gamma_{w}$ for absorption due to the external source.

The atomic system is represented by a two-level system, i.e. the $3 S$ - and $3 P$-levels, with population densities $n_{1}$ and $n_{2}$. respectively. The upper level is populated at the rate $\left(\Gamma_{x^{\prime}}+\Gamma_{s}\right) n_{1}$ and depopulation is assumed to be by spontaneous emission and collisional deexcitation at rates of $A n_{2}$ and $k n_{2}$, respectively, where $A=$ Einstein coefficient for spontaneous emission and $k=$ quenching rate constant. In the stationary state, one has

$$
n_{2} / n_{1}=\left(\Gamma_{n}+\Gamma_{i}\right)(A+k)
$$

if no saturation effects are present; the latter assumption follows from the experimental fact that the $3 P-3 S$ fluorescence resulting from one-photon wing excitation of the $3 S-3 P$ transition proved to be a linear function of the laser power.

The number of transitions per second within the volume $V$ due to reabsorption of spontaneous emission is

$$
\Gamma_{s} n_{1} V=A n_{2} V \xi \text {, }
$$

where

$$
\xi=\overline{k(\lambda)} l_{\text {ef }} .
$$

It follows that

$$
\Gamma_{s}=A n_{2} \xi / n_{1},
$$

$1-\xi$ is interpreted as the escape probability from the volume $V$ when $\xi \ll 1 ;^{(26)} \overline{k(\lambda)}=$ absorption coefficient as averaged over the absorption line profile; $l_{\mathrm{ef}}$ is a characteristic dimension of the volume $V$ (average length of escape path for photons). From Eq. (Al), we note that

$$
A n_{2}=Y\left(\Gamma_{w^{\prime}}+\Gamma_{5}\right) n_{1}
$$

where $Y=A /(A+k)$ is the efficiency of fluorescence. From Eqs. (A4) and (A5), we obtain, since $\xi Y \ll 1$,

$$
\Gamma_{s} / \Gamma_{w} \approx \xi Y \text {. }
$$

This ratio is independent of the detuning and the intensity of the laser beam. The quantity $\xi=\overline{k(\lambda)} l_{\text {eff }}$ can be found from the curve-of-growth as the relative deviation of the curve from the initial asymptote at the concentration $\left(l_{\text {eff }} / R\right) c$, where $c=$ concentration of metal solution giving rise to the ground-state density $n_{1}$ and $R=$ path length of the observed ffuorescence beam within the flame; $R$ is about equal to the radius of the cylindrical flame. We have made use of the fact that the Na-concentration inside the flame is uniform. From a curve-of-growth of the thermal emission of the $3 P-3 S$ fluorescence of sodium, we obtain for $c=2560 \mathrm{ppm}, l_{\mathrm{eff}} \approx 100 \mu \mathrm{m}, R=7.5 \mathrm{~mm}$ and $Y=0.67$ a value of $\xi Y=0.1$ 